Estudios Románicos, Volumen 27, 2018, pp. 33-48

ISSN: 0210-491

eISSN: 1989-614X

DOI: https://doi.org/10.6018/ER/346521

\title{
LA IDENTIDAD FEMINISTA EN LA OBRA DE CARMEN DE BURGOS
}

(Feminist Identity in the Work of Carmen de Burgos)

\author{
Anja Louis* \\ Sheffield Hallam University. Reino Unido
}

\begin{abstract}
Carmen de Burgos is one of the most important Spanish feminist writers and activists of the twentieth century. Her career is particularly interesting since she uses her writing for political purposes at a historical time when the increase in literacy rate resulted in an unprecedented demand for reading material. This article fills a significant gap in Burgos Studies: its analysis of La entrometida/The Busybody shows how Carmen de Burgos reworks popular fiction in order to foreground and question accepted identity formations. The discussion focusses on happiness as well as the social function and political value of popular culture, in particular the symbolic role played by a fictional feminist.
\end{abstract}

Keywords: De Burgos; First-wave feminism; Melodrama; happiness; Spanish popular culture; La entrometida/The Busybody; La mujer moderna y sus derechos/The Modern Woman and her Rights.

Resumen: Carmen de Burgos, escritora y activista política, era una de las más importantes defensoras del feminismo del siglo XX. Su trayectoria es de sumo interés, porque combinaba la profesión de periodista y escritora con una agenda política. Usaba la cultura como vehículo de propaganda feminista durante una época con creciente demanda de un pueblo cada vez más alfabetizado. Este artículo llena una importante laguna en los Estudios de Carmen de Burgos: A través de la novela corta La entrometida se analiza la manera cómo De Burgos destaca y cuestiona la formación de identidades. Se enfoca en el tema de la felicidad y la función social y política de la cultura popular, en particular el papel simbólico de una feminista ficticia.

Palabras clave: De Burgos; Feminismo de primera ola; Melodrama; Felicidad; Cultura popular; La entrometida, La mujer moderna y sus derechos.

${ }^{*}$ Dirección para correspondencia: Dra Anja Louis, Languages and Cultures, Sheffield Hallam University, Stoddart Building, Sheffield S1 1WB, Reino Unido (a.louis@shu.ac.uk) 


\section{Introducción}

Carmen de Burgos (1867-1932), escritora y activista política, era una de las más importantes defensoras del feminismo a principios del siglo XX. Conocida como la divorciadora y la dama roja en su ciudad adoptiva de Madrid, fue testigo de la transición de un sistema monárquico a la proclamación de la Segunda República, una época en la que España podía alardear de tener una de las democracias más modernas en la Europa del momento. Ella era una "creación" de su época y en su historia personal se ven reflejadas la modernización y la democratización de España, un proceso que llevó al país a implementar reformas sociales y políticas sin precedentes. Puede ser considerada como una especie de puente entre la generación de feministas del siglo XIX, como por ejemplo Concepción Arenal (1820-1893), Concepción Gimeno de Flaquer (1850-1919) y Emilia Pardo Bazán (1851-1921), y sus contemporáneas como Clara Campoamor (1888-1972), Margarita Nelken (1894-1968), Federica Montseny (1905-1994), Victoria Kent (1891-1987) y María de la O Lejárraga (1874-1974). Gracias a los esfuerzos incansables de académicos tanto en Europa como en los EE. UU. durante los últimos cuarenta años, por fin De Burgos ha ido ganando el reconocimiento que merecía como escritora, feminista y activista. Como consecuencia de este esfuerzo conjunto, hoy en día se le considera una figura clave de la España de principios del siglo XX. Cuando empezó este 'trabajo de rescate', ninguno de los que participábamos nos podríamos imaginar el impulso que cogería. Hemos pasado del olvido a un punto en el que De Burgos se ha convertido en una referencia obligada en los estudios de género en Europa.

Maestra de profesión y periodista de vocación, en 1903 obtuvo su primer puesto como redactora en el recién fundado periódico progresista el Diario Universal, siendo la primera mujer en España que formó parte de una redacción. En 1909, durante la guerra de África, se convirtió también en la primera mujer en ejercer una corresponsalía de guerra y causó gran revuelo con sus reportajes sobre lo absurdo de la guerra y las innecesarias matanzas. Su columna diaria (casi siempre en portada) pretendía informar a las lectoras tanto sobre los temas tradicionalmente considerados femeninos -la moda, la salud o la costura-como lo que hoy en día se entiende por lo "políticamente correcto". Así, De Burgos usó su columna "para mujeres", y aparentemente tradicional, para diseminar ideas políticas como la defensa de los sefardíes, la abolición de la pena de muerte, los derechos del niño y de la clase trabajadora y, sobre todo, los derechos de la mujer. Escribió miles de artículos para numerosos periódicos (Diario Universal, $A B C$, El Globo, Heraldo de Madrid, Nuevo Mundo y Prometeo, entre otros) a lo largo de su vida, logrando fama bajo el seudónimo Colombine.

La reivindicación social estaba inscrita en el ADN de Carmen de Burgos. El caso es sumamente interesante porque ella, como pocas mujeres de su época, combinaba la profesión de periodista y escritora con una agenda política. Usaba primero el periodismo y luego la literatura como vehículos de propaganda feminista. En 1903, De Burgos realiza una encuesta sobre el polémico tema del divorcio en una época en que éste ni siquiera existía: sólo la separación estaba contemplada dentro de la ley. La prensa reaccionaria, indignada por su audacia, le ataca ferozmente y le da el apelativo de "la 
divorciadora". En 1906, repite su éxito en el debate público con un tema no menos controvertido: el sufragio femenino. Las dos encuestas causan tanto revuelo que le enseñan cómo usar los medios de comunicación en beneficio propio. Tiene la suerte de poder participar en uno de los fenómenos editoriales con más éxito, la llamada Promoción de El Cuento Semanal (Magnien 1986, Pujante Segura 2014). En esta serie publicará sus primeras cuatro novelas cortas. Debido a la creciente demanda de un pueblo cada vez más alfabetizado, la publicación de novelas cortas en un formato semanal gozaba de gran éxito, alcanzando tiradas de entre 50000 y 200000 ejemplares. Le dio la oportunidad de divulgar sus ideas feministas en un medio literario de masas.

\subsection{Melodrama}

Para apreciar la obra de Carmen de Burgos en su totalidad nos pueden servir de ayuda dos debates críticos que se han popularizado mucho bajo la disciplina de los estudios culturales en el mundo anglosajón: primero, la discusión sobre la dicotomía entre la cultura de masas y la cultura de élites -con la intención de rescatar la primera-; y segundo, la actitud de juzgar el valor de una obra literaria no tanto por su contenido estético como por su función de vehículo para diseminar ideas políticas -en este caso el melodrama-. Por tanto, quizás no sea de extrañar que el mundo angloparlante tenga mucho más aprecio a la obra de Carmen de Burgos que su país natal. Su obra feminista puede considerarse una campaña de concienciación, especialmente si tomamos en cuenta sus ensayos feministas. Andreas Huyssen en su libro After the Great Divide (1986) analiza la dicotomía entre la cultura de masas y la alta cultura burguesa. Según él, la 'ansiedad de contaminar' la cultura de élites con la cultura de masas devino en una canonización de los textos modernistas y el olvido sistemático de formas de cultura no legítimas. Esa contaminación percibida venía en parte del hecho de que los lectores pertenecían a un grupo social nuevo y sin 'cultura': la clase baja y, además, femenina. En el caso de nuestra autora hay dos razones para el menosprecio: primero, el periodo creativo de De Burgos coincide con las generaciones del 98 y del 27, la llamada Edad de Plata, y por lo tanto el enfoque y el aprecio de toda la crítica literaria se concentra en esos autores. Segundo, la historiografía oficial del franquismo borraba tanto a feministas como a socialistas de la memoria nacional.

Además, en sus novelas cortas aprovechó al máximo el género del melodrama. Una mirada al desarrollo histórico del género pone de relieve su potencial revolucionario. Por ejemplo, en la literatura de izquierdas este potencial se ha usado para agitar las masas e incitar la rebelión. El enfoque al grupo social de los desamparados (en el melodrama de las revoluciones francesa o rusa, por ejemplo) da lugar a una identificación de los lectores con su héroe/heroína (Gerould 1994: 185). La historia del melodrama también incluye ejemplos más conservadores, como la ficción popular del siglo XIX o su reciclaje en el cine clásico de Hollywood de los años 40. En ambos casos los valores burgueses exigen un papel femenino tradicional del ángel del hogar. Lo interesante de la ficción de nuestra autora es que pone de relieve un espacio interpretativo subversivo que va en contra del supuesto valor conservador del texto. Es importante reconocer que justamente por ser un texto tradicional es un mecanismo potente de crítica y cambio 
social, como una especie de "caballo de Troya". De Burgos sacó partido de este género subversivo para divulgar su crítica del mundo patriarcal. Usando la retórica y la visión melodramática del mundo podía criticar fácilmente una realidad legal y social que para las mujeres era tan maniquea como la que pintaba en su ficción. La situación legal en esta época era tan injustamente favorable al hombre que no era difícil para De Burgos imitar una realidad que a menudo alcanzaba ribetes de melodrama.

Sin embargo, según muchos críticos literarios, su melodrama relata historias obvias, mal narradas y con poca destreza literaria. Peter Brooks, en su libro pionero y seminal The Melodramatic Imagination (1995), explica que las características del melodrama (emociones extremas, coincidencias improbables, compresión de tiempo, un deseo de decirlo todo) se han entendido mal y demuestran una falta completa de compresión de las premisas del género:

What we most retain from any consideration of melodramatic structures is the sense of fundamental bipolar contrast and clash. The world according to melodrama is built on an irreducible Manichaeism, the conflict of good and evil as opposites not subject to compromise. Melodramatic dilemmas and choices are constructed on the either/or in its extreme form as the all-or-nothing. Polarization is both horizontal and vertical: characters represent extremes, passing from heights to depths, or the reverse, almost instantaneously. The middle ground and the middle condition are excluded (Brooks 1995: 36).

Las mujeres de De Burgos no se quedan mudas, manifiestan sus opiniones (y oposiciones) con claridad dramática. Así el maniqueísmo melodramático y los epítetos morales sirven de fácil medio de comunicación: la lectora puede tomar partido y aceptar su credo. De allí su uso frecuente de hipérbole, antítesis y oxímoron -prueba del rechazo de matices y de su modus operandi de bipolaridad-(Brooks 1995: 40).

\section{Las múltiples mujeres modernas de Carmen de Burgos}

La modernidad supuso un reposicionamiento de la mujer en la sociedad: en Europa con la industrialización y la urbanización los movimientos feministas se iban consolidando al tiempo que las mujeres solteras de clase media empezaban a exigir la entrada a las profesiones, lo cual les garantizaría un sueldo correspondiente al nivel social de su familia (Evans 1977: 23-24). Por toda Europa las mujeres de clase media se iban dando cuenta de que se les negaban unos derechos civiles básicos. Se las trataba como menores ante la Ley, no tenían derechos de propiedad y no se les permitía trabajar. Evans señala que:

The aims of feminist movements were initially primarily economic in character. The early feminists demanded access for unmarried women to the professions and the right of married women to control their own property. They backed these demands by fighting for improvements in girls' secondary education and the admission of 
women to universities, in order to secure the qualifications necessary for engagement in professional activities and to attain the level of education necessary to manage their own domestic and financial affairs. As these demands were conceded, [...] middle-class women began to move into professions, above all into teaching (Evans 1977: 232).

La educación se consideraba una herramienta esencial para transformar la vida de la mujer ya que le brindaría la oportunidad de conseguir las cualificaciones necesarias para entrar en las profesiones y saber manejar sus asuntos domésticos y económicos. La intención era ser independiente, pero la emancipación de la mujer requería no solo acceso a la educación sino una profunda reforma del sistema jurídico ${ }^{1}$.

España a menudo se le considera "el vecino pobre" cuando se compara con otras democracias del mundo occidental y sus movimientos feministas no representan una excepción a esta norma. Los académicos han sostenido que el feminismo llegó tarde a España debido a la influencia limitada de la Ilustración en España y la reacción del catolicismo imperante contra los ideales igualitarios de la Revolución francesa (Scanlon 1986, Folguera Crespo 1997). Además, al igual que sus compañeras internacionales, las feministas españolas nunca llegaron a ponerse de acuerdo sobre una meta común ya que existían demasiadas facciones del feminismo: el feminismo católico, el moderado o el radical, por dar solo tres ejemplos. Para el feminismo católico la función principal de la mujer era la maternidad, mientras que las otras dos facciones luchaban, en distintas medidas, por la igualdad de derechos (Folguera Crespo 1997: 487). Independientemente del éxito o el alcance de los movimientos feministas en España, la obra de Carmen de Burgos demuestra claramente que existen suficientes semejanzas con otros movimientos feministas para poder ubicar a España dentro del llamado feminismo de la primera ola (first wave feminism). De Burgos sabía de sobra que en otros países europeos y en los EE. UU. la condición de la mujer no era mucho mejor que en España: las mujeres eran marginalizadas de forma social, cultural y jurídica, y como consecuencia cualquier tipo de activismo feminista resultaba muy innovador. Las feministas de la primera ola estaban convencidas de que el sufragio lo iba a cambiar todo, que conseguir el voto supondría la apertura de la vida pública para ellas y que el feminismo era sólo un mal necesario y, sobre todo, transitorio para lograr la igualdad entre los sexos. Cien años después sabemos que la igualdad está aún por alcanzar. Persisten los malos tratos: las noticias de agresiones a mujeres que leemos en la prensa de hoy tienen los mismos rasgos que en la narrativa de Carmen de Burgos². Continúa la discriminación salarial, hay una falta de representación femenina en la vida pública, y existe una tendencia a la profanación de lo femenino y la adulación de lo masculino. Es inquietante que la vida y la obra de Carmen de Burgos sigan tan relevante hoy en día.

Al evaluar la conceptualización de la mujer moderna por parte de Carmen de Burgos, es importante recordar el debate enraizado que existía dentro del pensamiento

\footnotetext{
$1 \quad$ Véase Enríquez de Salamanca (1992: 219-247), donde explica la subjetividad legal de la mujer en el discurso liberal y, entre otros mecanismos legales, la «discriminación por razón de sexo〉 además de la 'discriminación por razón de matrimonio'.

2 Véase por ejemplo La malcasada, El artículo 438, El hombre negro.
} 
feminista de 'igualdad' contra 'diferencia's . Estos conceptos opuestos habían resultado problemáticos para las políticas feministas desde el nacimiento del liberalismo. Para poder mantener la exclusión de las mujeres de la vida pública en una época en la que ellas se iban haciendo más activas políticamente, el patriarcado necesitaba asegurarse de que las mujeres se quedaran dentro de la esfera privada. Esto se consiguió gracias a la manipulación jurídica y cultural -lo último principalmente con el discurso del ángel del hogar-. Como señala De Burgos:

Se proclamó con todos los tonos patéticos que la naturaleza marca la misión de los dos sexos: El hombre debe trabajar, la mujer no debía ser más que madre, ángel del hogar, reunión de todas las gracias y bellezas. Esto traducido al lenguaje vulgar, significa que la mujer no debía ser más que servidora y recreo del hombre. Pero si se hubiera hecho una ley de acuerdo con su canto lírico, para que todos los hombres hubiesen tenido la obligación de sustentar a ese "ángel del hogar", al que ellos se encargaban de cortar las alas, sin que tuviesen necesidad de trabajar y sin menoscabo de su dignidad de mujeres, la protesta hubiera sido general. Invocar la maternidad para mantener la esclavitud envuelve un cinismo superlativo y un desconocimiento inexplicable de la expansión que requiere la actividad de las mujeres que no han sido madres y de las viudas y casadas que, después de criar y educar a sus hijos, terminada la misión materna, tienen energías que reclaman aplicación (Burgos 1927: 13)4.

A pesar de esa condenación del concepto de ángel del hogar, un análisis de la totalidad de los ensayos feministas de la autora muestra que De Burgos oscila entre su auto-declarado feminismo de igualdad y su discurso de diferencia, el cual conllevaba el riesgo de hacer el juego al dominante discurso masculino de diferencia, que clasificaba a las mujeres como inferiores. De manera parecida, el feminismo británico de los años 20 y 30 , por ejemplo, se caracterizaba por "a nervous hesitation between equality and difference, or a search for the fragile median position which saw women as «different but equal»" (Riley 1988: 62).

A veces De Burgos parece reforzar los argumentos patriarcales cuando reconoce que el papel social más importante de la mujer es la maternidad. La maternidad le concede a la mujer una superioridad moral y el uso del argumento de esta supuesta superioridad moral no era infrecuente en la lucha por el sufragio femenino. Durante mucho tiempo la idea de que el comportamiento moral de la mujer era superior al del hombre había estado presente en la propaganda feminista. En las campañas para conseguir el voto de

3 En el feminismo el discurso de la diferencia tiene dos aceptaciones: primero, de manera positiva se refiere a la diferencia biológica y fisiológica tanto como a una experiencia diferente en los ámbitos público y privado. Segundo, de manera negativa, se refiere a una categorización de inferioridad, exclusión y subordinación.

$4 \quad$ Véase también Enríquez de Salamanca: 'Uno de los principales cambios que introdujo el mundo liberal fue un nuevo discurso de género sexual que prescribió al ángel del hogar como modelo de mujer. Este modelo radica en la creencia en una diferencia básica entre los sexos, una diferencia que parte de la fisiología e incluye lo mental y sentimental; se abandona por tanto el antiguo principio de que la mujer es una imitación defectuosa del varón para proponer a ésta como un ser diferente y complementario del hombre' (Enríquez de Salamanca 1998: 219). 
la mujer en muchos países se recurría al argumento de que la mujer podría contribuir a refrenar la inmoralidad y el desorden en la sociedad (Evans 1977: 233). Motivada por el pragmatismo político, De Burgos utilizaba tácticas parecidas en su propia campaña para conseguir el sufragio femenino. La cuestión para De Burgos no era que el cuerpo de la mujer fuera superior gracias a su función reproductiva; más bien era la idea de que el papel social de la mujer como madre le hacía superior moralmente, ya que las madres eran más altruistas. El sufragio no sólo era una ventaja para las mujeres en general sino para las madres en particular porque significaba que podrían educar mejor a sus hijos. Una vez más la maternidad era el factor diferenciador y un argumento que utilizaban ambos lados del debate: para el discurso dominante masculino la maternidad servía para establecer el concepto del ángel del hogar que justificaba que las mujeres no salieran de la esfera privada, mientras que el discurso feminista de igualdad (lo normal en la primera ola) utilizaba la maternidad como prueba para sostener que la inclusión de la mujer en el mundo público sería positivo para la sociedad. Hoy en día todavía se escucha este argumento con los debates sobre el papel afectivo de la mujer y cómo esto podría cambiar el tejido social. Sin embargo, el uso de argumentos de la superioridad moral concedida por la maternidad y las diferencias biológicas, en un momento histórico en el que la mujer aún no disfrutaba de la igualdad jurídica, se podría considerar una táctica cuestionable ya que podría hacer el juego a la oposición. Si examinamos su obra y su vida está claro que De Burgos encarnaba las tensiones entre la tradición y la modernidad, y en sus escritos aparecen múltiples representaciones de la condición femenina. En una época en la que la modernidad era incipiente, en sus escritos reflexionaba sobre mujeres que rompían las normas (por ejemplo, las viajeras, las feministas, las lesbianas, las mujeres en zonas de combate) y mujeres que reivindicaban una feminidad moderna en la que los esfuerzos de modernización se lograrían a través de la economía doméstica y la maternidad. La lucha feminista de Carmen de Burgos duró toda su vida. El futuro mejor que se imaginaba para las mujeres era igualdad de oportunidades e igualdad de estatus social, indiferente de las aportaciones sociales que añade la mujer. De allí su visión de múltiples modernidades.

\subsection{En busca de la felicidad feminista: La entrometida (1921)}

Podría parecernos extraño hablar de la obra de Carmen de Burgos en términos de la felicidad. Carmen de Burgos escribía, principalmente, sobre mujeres que son víctimas inocentes de un patriarcado hostil. La felicidad de la mujer brilla por su ausencia y la 'felicidad femenina' podría parecernos casi una contradicción de términos. Sara Ahmed (2010: 572) se pregunta: "What would it mean to suspend belief that happiness is what we wish [...] or even that happiness is a good thing?". ¿Hasta qué punto era posible la felicidad para una mujer feminista a principios del siglo XX en España? ¿A qué se parecería la historia de una feminista feliz? A las académicas feministas como Mary Eagleton y otras les gusta leer textos llenos de ambigüedades que están abiertos a múltiples interpretaciones (Eagleton 2011: 178), advirtiéndonos que en la cultura popular, al igual que en la vida, las mujeres fuertes como ejemplos a seguir no sirven, porque representan un modelo que no permite momentos de debilidad y, por consiguiente, quizás las mujeres se sientan inadecuadas en comparación (Eagleton 2011: 183). Sin embargo, 
creo que todas buscamos modelos que nos inspiren; de hecho, para los movimientos feministas la búsqueda de mujeres que hayan conseguido romper el techo de cristal sigue vigente. Siempre que seamos conscientes de las dificultades que presenta una visión sentimentalista del feminismo, dar modelos positivos a las mujeres es fundamental.

Durante siglos las feministas han demostrado cómo los deseos y las necesidades de las mujeres han sido ignorados por el patriarcado, que ha convencido a las mujeres de que la felicidad yace en la felicidad de otros (padres, maridos, hijos) y, por lo tanto, en la abnegación femenina. Una ética de abnegación o sacrificio personal está directamente en conflicto con el concepto de los derechos que ha apoyado la reivindicación de la mujer a la justicia social ${ }^{5}$. Como veremos, la protagonista de La entrometida sacrifica su propia felicidad para conseguir una sociedad en la que todas las mujeres puedan disfrutar de unos derechos básicos, como la posibilidad de elegir su propio camino y el desarrollo personal. Las críticas feministas siempre han notado una contradicción entre las atrevidas demandas que Carmen de Burgos presenta en el ensayo más exhaustivo y teórico La mujer moderna y sus derechos (1927) y la falta de ejemplos de mujeres emancipadas que pudieran llegar a inspirar a sus lectoras en su obra narrativa. $L a$ mujer moderna ubica a la escritora dentro del feminismo de la primera ola en cuanto a su postura política y moral. De Burgos deconstruye los discursos patriarcales dominantes, mostrando la arbitrariedad de sus construcciones y el egocentrismo del derecho falocrático. La mujer moderna y sus derechos puede considerarse una obra precursora del famoso Le deuxième sex de Simone de Beauvoir, que unos veinte años después se convirtió en lectura obligada para las feministas de la segunda ola. La mujer moder$n a$ (1927) analiza detalladamente el derecho positivo en contra de la mujer y, sobre todo, el razonamiento en el que se basan las leyes para legitimar el trato a las mujeres como eternas menores de edad. El tratado demuestra cómo el discurso legal dominante de la época construye razones -supuestamente biológicas- para demostrar que las mujeres son, verdaderamente, inferiores, y de allí la justificación de la discriminación por razón de sexo. Después de mostrar la arbitrariedad del razonamiento masculino, De Burgos enumera los derechos más importantes para el progreso de la mujer: derecho a saber, derecho al trabajo, igualdad dentro del matrimonio, derechos de las madres, derechos políticos. A partir de una enumeración de derechos civiles y políticos De Burgos construye su visión de la mujer moderna para quien la felicidad es un sine qua non. Explícitamente relaciona la felicidad a los derechos de la mujer. Aunque un breve análisis de La mujer moderna muestra solamente 24 entradas de las palabras 'felicidad' o 'in/feliz' ${ }^{6}$ en toda la obra (320 páginas), es interesante centrarse en los temas: es obvio que el matrimonio y el divorcio son de suma importancia en la obra de la autora (46, $137,141,194)$. También se hace referencia a la felicidad privada $(47,75,131,134,258)$, no tanto como 'ángel del hogar' sino como 'felicidad en el hogar'. De Burgos también

$5 \quad$ Como explica Carol Gilligan (1993: 132): 'The notion that virtue for women lies in self-sacrifice has complicated the course of women's development by pitting the moral issue of goodness against the adult questions of responsibility and choice.'

6 Véase La mujer moderna, pp.12, 46, 47, 52, 61, 75, 94, 121, 129, 130, 131, 134, 137, 138, 141, 177, 187 , 194, 200, 258, 274, 278, 280. 
relaciona la felicidad a los derechos de igualdad y justicia social para las mujeres (121, 134, 274). Además, relaciona la justicia social con la felicidad de una nación entera: "[L]a felicidad de las naciones está en razón directa de la suma de justicia que distribuyan entre los individuos que las forman." (12) Así se puede considerar una precursora de los estudios de la felicidad (Happiness Studies ${ }^{7}$ ) que han surgido recientemente. La primera ola del feminismo español reivindica la búsqueda a la felicidad y el bienestar como derecho universal incluyendo a las mujeres en esa fórmula legal. Sin embargo, y para la consternación de las académicas, en sus novelas y cuentos esta teórica mujer nueva y feliz que De Burgos construye en su ensayo brilla por su ausencia.

Aunque poco conocida, La entrometida es una obra maestra dentro de la narrativa de Carmen de Burgos, no solo porque representa una interpretación magistral de los debates feministas de la época, sino porque cuenta la historia de las luchas y las dificultades a las que se enfrenta una feminista de la primera ola. ${ }^{8}$ Una activista feminista escribe sobre una activista ficticia para cuestionar la aceptada formación de identidad. Esta novela corta demuestra las complicaciones del activismo político, y aún más importante, el coste personal que puede llegar a tener. Clarisa encarna el feminismo al que aspira la obra teórica de nuestra autora. La entrometida es la historia de una mujer joven y soltera que lleva una vida independiente. ${ }^{9}$ Tiene 25 años, es culta, ha viajado por el mundo y, al expresar sus opiniones e ideas, es franca y abierta. Es activa políticamente y no esconde sus ideales feministas:

Trabajaba por sus ideales de un modo incansable. Había visitado a todos los ministros, no faltaba un día al Congreso y al Senado, traía revuelto el Instituto de Reformas Sociales pidiendo datos de cuestiones femeninas. No dejaba vivir al rector de la Universidad ni a los directores de los Centros que de ella dependen, presentando a su estudio vastos planes de reformas pedagógicas y de instituciones que era preciso crear. [...]. Estaba en todo y en todas partes. (6)

La clave de la narración es la tensión entre el feminizado Pérez Blanco, bon vivant y caballero de clase media, y la masculinizada Clarisa, la codependencia entre ambos, su admiración mutua y su amor latente. Hasta cierto punto son almas gemelas: los dos están atrapados dentro del dominante discurso de género de su época y los dos sufren por ello; comparten el narcisismo y la independencia de los de fuera y, como consecuencia, no es sorprendente que se encuentren más cómodos con el sexo opuesto.

$7 \quad$ Véase por ejemplo Csíkszentmihályi, Mihály. Flow: The Psychology of Happiness. London: Rider, 1992; McDahon, Darrin M (2006): Happiness: A History. New York: Grove Press. También Journal of Happiness Studies http://www.springer.com/social+sciences/wellbeing+\%26+quality-of-life/journal/10902

8 Véase Núñez Rey (2005: 506-507), donde explica que La entrometida se publicó el mismo año que De Burgos, como presidenta de la Cruzada de Mujeres Españolas, organizó la primera manifestación a favor del sufragio. El Heraldo de Madrid anunció: 'es el amanecer de un serio movimiento feminista, y este primer acto de las sufragistas españolas sorprendió a los diputados.'

$9 \quad$ Al lector culto el nombre le recuerda a la novela Clarissa de Samuel Richardson (1748), traducido al español en 1829. Es dudoso que las lectoras de De Burgos hubieran leído la novela. Sin embargo, la autora misma hace una referencia intertextual intencionada. 
Sara Ahmed, en su artículo "Killing Joy: Feminism and the History of Happiness" (2010), reflexiona sobre aquellas mujeres que son consideradas agitadoras y aguafiestas que estropean la felicidad. Clarisa es una agitadora: su lucha política en parte proviene de un auténtico deseo de mejorar el futuro de las mujeres por la participación activa y pasiva en la vida pública (6) y a través de acciones concretas como construir escuelas y centros hospitalarios para las mujeres (4). Su aspiración de formar parte de la clase política también está alimentada por la vanidad, la posibilidad de coincidir y codearse con personajes importantes: "«Ramón y Cajal me ha dicho que es un feminista convencido», «Azorín está encantado de mis proyectos» [...], «Eso me recomendó Luca de Tena»" (13). Como mujer activa e independiente, la felicidad de Clarisa también proviene de una sensación de superioridad. En este sentido su felicidad temporal es una consecuencia de la satisfacción que le aporta su activismo político y su vida independiente, hasta el momento en el que la cruda realidad la despierta de forma brusca. En marcado contraste con la mayoría de las mujeres en este relato, el fuerte deseo de Clarisa de ser independiente la convierte en una autoproclamada luchadora feminista. Sin embargo, el resultado de su superioridad y sus delirios de grandeza es una conceptualización idealizada del activismo político. De hecho, la novela corta se acerca peligrosamente a la novela sentimental, tanto en su estructura narrativa como en su lenguaje: el compañerismo inocuo de Clarisa y Pérez Blanco quien, no obstante, es su protector masculino ("salió tras de Clarisa en su papel de escudero de la Princesa" (9)) y su feminismo de fantasía que no llega a dar resultados.

Tanto Jo Labanyi (2004) como Sonia Núñez Puente (2008) han escrito de manera convincente sobre las novelas sentimentales en español y la construcción de discursos de feminidad. En sus análisis de este tipo de novela durante los primeros años de la época franquista, subrayan cómo los textos románticos se pueden considerar un ejemplo de una "modernidad conservadora" 10 por su representación de protagonistas femeninas que son activas y que van ascendiendo la escala social. A pesar del final feliz conservador en el que la protagonista siempre acaba casándose con el héroe, las heroínas llevan unas vidas muy activas e independientes. Las protagonistas están representadas como independientes y por lo tanto subvierten la ideología del régimen. Como Núñez Puente demuestra:

Romance novel discourse actually appears to be relatively resistant to the hegemonic power that supports it. The protagonists of the novels [...] are characters constructed as resistant women, something that ends up affecting the female readers themselves as consumers, who find an alternative world in the fiction that they can make their own. It therefore becomes necessary to examine the processes that govern the production of popular culture and that transform, in neo-Gramscian terms, the high culture/popular culture dichotomy, to ultimately end up placing woman and the concept of woman outside the boundaries of cultural reception. (Núñez Puente 2008: 228)

10 Haciendo referencia al concepto que propone Michael Richards en Un tiempo en silencio. La guerra civil y la cultura de la represión en la época de Franco (2006). 
Aunque en los años veinte España se encontraba al borde de una nueva época, los discursos jurídicos y culturales imperantes aún mantenían a las mujeres en una posición inferior y, por lo tanto, se puede comparar este periodo con la situación que existía durante la época de Franco.

De Burgos coge la fórmula de la novela sentimental y la subvierte. Sus heroínas suelen ser jóvenes, inocentes y abnegadas pero, a diferencia de los personajes femeninos en los tradicionales relatos sentimentales (que siempre son mucho más afortunados que los personajes de nuestra autora), sus experiencias del cortejo acaban mal. ${ }^{11}$ Sin embargo, La entrometida es aún más compleja porque con los giros que da la trama se introducen dos objetos de deseo. Como en cualquier relato sentimental, el desarrollo de una relación entre los protagonistas es lo esencial de la historia, y hasta cierto punto la tensión dramática entre Clarisa y Pérez Blanco es el eje de la narrativa. Aun así, si en la ficción sentimental el objetivo final es casarse con un hombre, a nuestra heroína no le interesa mucho conocer a un hombre; en cambio, lo que ella busca es entrar en el mundo masculino con todas las libertades y los privilegios que ello conlleva. Se enamora de la política y vive en un mundo de fantasía que es igual de fantástico que el que habitan las mujeres en las tradicionales novelas románticas. En el peor de los casos, su 'feminismo de fantasía' es un mecanismo de evadir la realidad; en el mejor de los casos, el relato demuestra lo difícil que es lograr cambios sociales para las mujeres y por lo tanto la frustración de Clarisa ante esta dificultad se convierte en fantasía como mecanismo de asimilar su fracaso. No es que La entrometida sea una novela sentimental, sino que la búsqueda masculinizada de Clarisa por una identidad y un lugar en la sociedad tiene un marco romántico. Esta falsa conciencia de la felicidad en una lucha feminista nos recuerda a la (in)felicidad de las amas de casa. Tanto a las feministas como a las aburridas esposas de clase media les resulta demasiado doloroso examinar la realidad de sus respectivos encarcelamientos en un mundo falocrático. Como explica Ahmed:

There is something false about our consciousness of the world: we learn not to be conscious; we learn not to see what happens right in front of us. It is not that an individual person suffers from false consciousness but that we inherit a certain false consciousness when we learn to see and not to see things in a certain way. (Ahmed 2010: 590; cursiva mía)

Clarisa no hereda esta falsa conciencia de las 'amas de casa felices', pero utiliza la misma manera de enfrentarse a su situación o más bien evadirla. De hecho, podríamos decir que la raíz del problema de Clarisa yace en su retirada del mundo real al mundo de feminismo idealizado. La tensión entre su percepción acertada de la realidad y su visión romántica del feminismo es un tema recurrente en la obra. Dos veces $(11,17)$ un choque con la realidad la despierta de su mundo imaginario. Por ejemplo, su criada Juana le advierte de su precaria situación financiera: “¿Y con qué voy a preparar algo, señorita?" (11). Como resultado, reconoce que "el triunfo, el lado práctico de su labor,

11 Véase Louis (2002), Louis (2005) y Louis (2017). 
se hacía esperar demasiado" (11). Y, para colmo, su criada no entiende por qué el activismo feminista de Clarisa es tan importante:

-Pero, señorita, no se canse usted tanto, es usted joven, diviértase un poco, en lugar de andar siempre [...] de aquí para allá.”

-¿Tú qué sabes de eso, Juana? Vosotras no podéis comprender a lo que nos obliga la misión que hemos de cumplir para librarnos de la esclavitud a que todas las mujeres estamos condenadas. Se hace preciso luchar, reclamar nuestros derechos...salvarte a ti y a otras infelices como tú...

- Pues lo que es por mí no se apure la señorita, que yo estoy bien contenta así. (13)

Está claro que ni las mujeres de clase media ni las de clase obrera muestran interés en la lucha política de Clarisa. Las primeras se encuentran demasiado cómodas en su falsa conciencia y las últimas estarán demasiado ocupadas con llegar a fin de mes.

El primer amor de Clarisa -es decir, el feminismo- la acaba quemando y sus necesidades económicas requieren unas medidas drásticas. En un giro inexplicable de la trama se convierte de feminista a escritora de novelas rosas para ganar dinero. Se podría interpretar como una forma de prostitución intelectual en la que vende su alma en vez de su cuerpo; resulta apropiado que su novela inacabada se titule Memorias de un alma. Además, acepta la ayuda de Pérez Blanco para escribir una historia de amor:

La obra avanzaba. Clarisa contaba a Pérez Blanco las sensaciones íntimas que había experimentado y las que imaginaba, y él le daba la forma literaria, las impregnaba de su picardía de hombre. Todos los días iba a casa de Clarisa y le ayudaba en su trabajo, deleitándose al oír sus confesiones, como los viejos curas que tienen su deleite en el recuerdo. (19)

Con fidelidad a la formula romántica Clarisa en su debilidad se deja manipular por el héroe. Curiosamente el único modo de dominar a Clarisa es impregnar el texto más bien que el cuerpo femenino. Este giro narrativo entonces introduce novelas sentimentales en un segundo nivel: primero, Clarisa corteja el feminismo, y segundo, Clarisa se convierte en escritora de novelas rosas. La cruda realidad la despierta repentinamente de su feminismo de fantasía y, desgraciadamente, sus intentos de trabajar como escritora también fracasan.

¿Qué significa para la lectora de la obra? En este punto las expectativas de un final feliz para la heroína se han esfumado, pero la lectora que sigue las circunstancias de Clarisa tiene la ventaja de poder distanciarse del personaje. La lectora puede ser más lista que la heroína porque ella comprende la situación mientras que Clarisa aún anda a oscuras. No tiene interés en el amor y fracasa como activista, se retira a un mundo de feminismo de fantasía, un proceso que podemos interpretar tanto como una huida y una protesta contra las vicisitudes de la lucha política y la formación conservadora de género a la que las mujeres españolas tenían acceso. La historia de Clarisa, por tanto, responde a un doble impulso: alienta el deseo de las mujeres de atribuirse poder a la vez 
que contiene ese deseo al darlo una forma falocrática. Ann Snitow, al escribir sobre las novelas de Harlequin ${ }^{12}$, sostiene que la novela romántica le permite a la mujer el único momento de trascendencia que se acepta socialmente en el ciclo del cortejo:

When women try to fantasize about success, mastery, the society offers them one vision, the power to attract a man. [...] When women try to project a unique self, the society offers them very few attractive images. True completion for women is nearly always presented as social, domestic, sexual. One of the culture's most intense myths, the ideal of an individual who is brave and complete in isolation, is for men only. (Snitow: 2011: 138, cursiva mía)

Con determinación Clarisa se resiste a la tradicional identidad de género, lo cual explica la razón por la que al final acaba fracasando. Clarisa fantasea sobre el éxito del feminismo, pero termina siendo una mujer cuyo único poder es atraer a un viejo. Pérez Blanco rompe con ella por publicar su novela inacabada sin su permiso. Ahora desesperada por conseguir dinero, y con la consternación de las lectoras, está realmente a punto de prostituirse al pedir a un señor mayor que la apoye económicamente. Sintiendo compasión por ella, le compra un billete para viajar al extranjero. Este final es brusco y típico en la obra de Carmen de Burgos. Un tiempo después, no sabemos cuánto, Pérez Blanco sostiene que se ha olvidado de ella. Clarisa le escribe desde Londres diciéndole: "Prefiero quedarme en este país, donde todos los caminos están abiertos a la mujer. No sé aun lo que he de hacer; dudo si seguir en mi propaganda o si seguir sus consejos. Estoy indecisa entre meterme a escritora o meterme a cocota" (22). Esta última frase de la obra nos deja a nosotras decidir el porvenir de Clarisa en Londres.

\section{Conclusión}

Parece que a la lectora se le anima a ver múltiples lecturas de la obra: durante la historia puede identificarse o con la nueva mujer independiente o con la mujer que se rinde y huye a Londres, o con ambas ${ }^{13}$. En las historias románticas tradicionales el final feliz es el matrimonio, pero el final de La entrometida está abierto a múltiples interpretaciones. ¿Hasta qué punto tiene importancia un final feliz feminista? ¿Puede perjudicar al mensaje feminista un final ambiguo? Una noción romántica de la felicidad se podría, una vez más, leer como una medida de control patriarcal. Clarisa se construye como una mujer resistente: se resiste a los ideales hegemónicos masculinos de la felicidad femenina y, sin embargo, ella fracasa en su propio intento de encontrar la felicidad. A pesar de ello, se mueve libremente dentro de la esfera pública cuando lucha por los mismos derechos para sus compañeras sufridoras. Para Clarisa, la autodeterminación no es posible en España, tiene que emigrar a Londres, lo que no representa un final

12 Novelas románticas.

13 Véase Labanyi (2004: 8) cuando explica que: 'Different readers are likely to have responded differently, but generally the reader seems to be invited to have it both ways: to identify with the modern, independent woman while simultaneously identifying with the woman who puts domesticity above everything' 
feliz pero sí optimista, hasta cierto punto, al menos para Clarisa. No obstante, también alude a un futuro sombrío para las que no se pueden huir a otro país. Es obvio decirlo, pero no es tanto un reproche al comportamiento de Clarisa, sino una crítica devastadora de la sociedad española de principios del siglo XX.

Una interpretación algo más optimista podría ser que las lectoras aprenden a través de la experiencia de Clarisa de su crisis y su llegada a un espacio más positivo. La cuestión crucial para determinar si la cultura tiene poder político y discursivo para el cambio social podría ser si la actuación segura y desenvuelta de Clarisa permanece en nuestras mentes; si es así se podría considerar un modelo a seguir. Es curioso que el conocido estudio de Radway $(1985)^{14}$ sobre la recepción por parte de las lectoras proponga una similar solución a medio camino, es decir, que la ficción romántica es una manera de enfrentarse a los problemas, porque da a las mujeres una manera de manejar su situación desfavorecida, aunque no proporcione una solución a los problemas en sí. La expectativa de un final feliz para la protagonista también implica un juicio moral acerca del bien y el mal: queremos que nuestra heroína sea recompensada por todo lo que ha sufrido. Un final apropiado para la lectora, entonces, podría ser simplemente que el personaje con el que se identifica esté contento. Un final no de 'felicidad para siempre', sino uno en el que las cosas no salen mal del todo para la heroína. Puede que no existiera un final feliz fácil y feminista que se podría imaginar en la España de principios del siglo XX. Aunque las discrepancias entre las exigencias feministas de sus ensayos y las representaciones de la mujer y la sociedad en su obra narrativa nos pueden llegar a incomodar, podría ser simplemente la tensión habitual entre la teoría y la práctica que todavía existe en los proyectos feministas hoy en día. La obra de De Burgos sigue siendo de suma importancia y se debe considerar lectura obligatoria en los institutos de España, si se quiere aprender de la historia.

\section{BIBLIOGRAFÍA}

AHMED, Sara (2010): "Killing Joy: Feminism and the History of Happiness", Signs. Vol. 35: 571-594.

BEAUVOIR, Simone de (1973 [1949]): Le deuxième sex. Paris: Gallimard.

BROOKS, Peter (1995): The Melodramatic Imagination. New Haven: Yale.

BURGOS, Carmen de (1916): El hombre negro (La Novela Corta, 8), Madrid: Prensa Popular.

(1921a): El artículo 438 (La Novela Semanal, 15), Madrid: Prensa Gráfica.

(1921b): La entrometida (La Novela Corta, 292). Madrid: Prensa Popular.

(1923): La malcasada, Valencia: Sempere.

(2007 [1927]): La mujer moderna y sus derechos. Madrid: Biblioteca Nueva.

CSÍKSZENTMIHÁLYI, Mihály (1992): Flow: The Psychology of Happiness. London: Rider.

EAGLETON, Mary ed. (2011): Feminist Literary Theory: a Reader. $3^{\text {rd }}$ ed. Oxford: Blackwell-Wiley.

Radway, Janice (1984). Véase también Modleski (1984). 
ENRÍQUEZ DE SALAMANCA, Cristina (1998): "La mujer en el discurso legal del liberalismo español". Catherine Jagoe, Alda Blanco y Cristina Enríquez de Salamanca eds., La mujer en los discursos de género, Barcelona: Icaria.

EVANS, Richard (1977): The Feminists: Women's Emancipation Movements in Europe, America andAustralasia 1840-1920. London: Croom Helm.

FOLGUERA CRESPO, Pilar (1997): 'Revolución y restauración. La emergencia de los primeros ideales emancipadores (1868-1931), Elisa Garrido González (ed.) Historia de las mujeres en España, Madrid: Síntesis, 451-492.

GEROULD, Daniel (1994): 'Melodrama and Revolution'. Jacky Bratton, Jim Cook and Christine Gledhill eds. Melodrama: Stage, Picture, Screen. London: BFI Publishing, 185-198.

GILLIGAN, Carol (1993): In a Different Voice: Psychological Theory and Women's Development. Cambridge: Harvard UP.

LABANYI, Jo (2004): "Romancing the Early Franco Regime: the Novelas Románticas of Concha Linares-Becerra and Luisa-María Linares" (Working Paper OP 13), Institute of European Studies, Berkeley: University of California.

LOUIS, Anja (2002): "Melodramatic Feminism: The Popular Fiction of Carmen de Burgos". Jo Labanyi ed., Constructing Identity in Contemporary Spain: Theoretical Debates and Cultural Practice. Oxford: Oxford UP.

(2005) Women and the Law: Carmen de Burgos, an Early Feminist. Woodbridge: Tamesis.

(2017) "In search of feminist happiness: Burgos's La entrometida". Louis, Anja, y Michelle Sharp eds., Multiple Modernities: Carmen de Burgos, Author and Activist. Abingdon-on-Thames: Routledge.

MAGNIEN, Brigitte, et al (1986): Ideología y texto en El Cuento Semanal (1907-1912), Madrid: Ediciones de la Torre.

MCMAHON, Darrin M (2006): Happiness: A History. New York: Grove Press.

MODLESKI, Tania (1984): Loving with a Vengeance: Mass-Produced Fantasies for Women. London: Routledge

NÚÑEZ PUENTE, Sonia (2008): "The Romance Novel and Popular Culture during the Early Franco Regime in Spain”, Journal of Gender Studies. Vol. 17.3: 225-236.

NÚÑEZ REY, Concepción (2005): Carmen de Burgos Colombine en la Edad de Plata de la literatura española. Sevilla: Fundación José Manuel Lara.

PUJANTE SEGURA, Carmen M. ${ }^{\mathrm{a}}$ (2014): De la novela corta y la nouvelle (19001950). Madrid: Síntesis.

RADWAY, Janice (1991 [1984]): Reading the Romance: Women, Patriarchy, and Popular Literature. Chapel Hill: University of North Carolina Press.

RICHARDS, Michael (2006): Un tiempo en silencio. La guerra civil y la cultura de la represión en la época de Franco. Barcelona: Crítica.

RICHARDSON, Samuel (1985 [1748]): Clarissa or the History of a Young Lady. London: Penguin.

(1829): Clara Harlowe, Madrid: Fuentenebro. 
RILEY, Denise (1988): 'Am I that Name?': Feminism and the Category of 'Women' in History, Basingstoke: Macmillan.

SCANLON, Geraldine M. (1986): La polémica feminista en la España contemporánea, 1868-1974, Madrid: Akal.

SNITOW, Ann (2011): "Mass Market Romance: Pornography for Women is Different", in Eagleton, Mary ed. (2011): Feminist Literary Theory: a Reader. $3^{\text {rd }}$ ed. Oxford: Blackwell-Wiley.

\section{PERFIL ACADÉMICO Y PROFESIONAL}

Profesora titular de Estudios Culturales e Interculturales (Universidad de Sheffield Hallam, Reino Unido). Se ha dedicado al campo interdisciplinario de 'derecho y cultura' en la cultura popular española (ficción, cine y televisión). Es autora de una gran variedad de publicaciones sobre Carmen de Burgos, entre ellos los libros siguientes: Women and the Law: Carmen de Burgos, an Early Feminist (2005) que es considerado un libro pionero en los Estudios de Género, mientras que el libro reciente Multiple Modernities: Carmen de Burgos, Author and Activist (2017) es una colección de ensayos por eminentes especialistas internacionales.

Fecha de recepción del artículo: 25-05-2018

Fecha de aceptación del artículo: 29-06-2018 